\title{
JUNTOS PARA ALCANZAR Y SUPERAR LAS METAS
}

El primer número de la Revista de la Sociedad Argentina de Diabetes (SAD) fue impreso en 1967, durante la presidencia del Dr. Néstor Serantes, 13 años después de la fundación de nuestra Sociedad. Hoy, a poco tiempo de cumplido el cincuentenario, nos encontramos en una Sociedad que con un número inicial de una decena de miembros llegó orgullosamente a su socio 2.700 , lo que nos obliga a situar a nuestra publicación a la altura de las circunstancias.

Las primeras palabras desde este Comité Editorial (CD) son de agradecimiento a la Dra. Carmen Mazza y al Prof. Manuel L. Martí, ambos Directores salientes, que con su profesionalismo, dedicación y sabiduría han dado soporte permanente y brillo a las tareas de publicación, y al Dr. Cristián Suárez Cordo, quien cumplió un rol destacado como Secretario de la Dirección. También, y en el mismo plano, es necesario agradecer en los nombres del Dr. León Litwak y el Prof. Pablo Arias a todos los miembros de los Comité que se sucedieron en estos últimos años y acompañaron con su tarea y opinión las modificaciones de fondo que fueron ocurriendo durante este tiempo y que queremos compartir.

Estas modificaciones, plasmadas en los últimos dos años pero cuyo origen se remonta a casi una década, tienen como aspectos más visibles el cambio de la compañía Editorial, un nuevo formato y la duplicación de la tirada de la edición normal de la Revista llevándola a 3.000 ejemplares, con una distribución postal más efectiva para alcanzar a los miembros de la SAD a nivel nacional e internacional. Con respecto a las ediciones especiales "XIX Congreso Argentino de Diabetes" y " $40^{\circ}$ Aniversario de la Escuela de Graduados SAD", se imprimieron 3.500 ejemplares de cada una (7.000 en total) que fueron distribuidos durante el Congreso mencionado en la ciudad de Mar del Plata. En consonancia con los requisitos que nos impone el crecimiento, desde el punto de vista de la gráfica, se ha logrado mejorar la calidad editorial, el diseño y la estética de la Revista.

En la actualidad, en relación al aspecto financiero, la edición, impresión y distribución de nues- tra publicación se autofinancian gracias a la venta de pautas publicitarias que incluyen medicamentos, alimentos y tecnologías médicas utilizadas para el tratamiento de la diabetes; asimismo dicho ingreso aporta un excedente que se destinará a otras actividades de promoción científica. Proyectamos, además de la mejorada edición en papel, un acceso directo a través de la web para los socios con cuota al día y disponer los números con más de dos años de antigüedad al acceso electrónico universal.

Con el fin de jerarquizar la Revista de la SAD entre las principales publicaciones científicas en español sobre diabetes mellitus estamos desarrollando un plan a mediano y largo plazo de cumplimiento de requisitos técnicos para lograr su indización en las principales bases de datos de revistas científicas nacionales e internacionales. En 2014 se incorporó la Revista a la base de datos LILACS (Literatura Latinoamericana y del Caribe en Ciencias de la Salud), coordinada por BIREME (Biblioteca Regional de Medicina, OPS/OMS), y se avanzó en el cumplimiento de requisitos de indización de las bases de datos coordinadas por el Centro Argentino de Información Científica y Tecnológica (CAICYT) del CONICET: Latindex, SciELO (Scientific Electronic Library Online) y Núcleo Básico de Revistas Científicas Argentinas. Actualmente estamos presentando la solicitud de indización de la Revista de la SAD en Latindex, para luego continuar con las dos siguientes. En el largo plazo, cumplidos los pasos anteriores, el objetivo es lograr la inclusión de la Revista en MEDLINE, la principal base de datos de citas bibliográficas biomédicas en Internet, que pertenece a la Biblioteca Nacional de Medicina de los Estados Unidos (NLM) y brinda acceso a las publicaciones periódicas biomédicas más importantes de todo el mundo.

Con el doble propósito de fortalecer la actividad de investigación y jerarquizar la Revista para que, a través de su indización, se transforme en un lugar cada vez más atractivo como medio de divulgación científica en el área de la diabetes para investigadores de Argentina y Latinoamérica, se ha estipulado en este año un cambio en el Comité Editorial, incor- 
porando representantes de los Comités de Trabajo de la SAD. Su función principal apunta al estímulo de la producción de trabajos de investigación y del aporte de material para su revisión por la Revista, originado tanto por su Comité de Trabajo como por miembros del mismo en forma individual o por profesionales no socios de la SAD con interés en dicha temática. Será además una tarea relevante a realizar por cada miembro designado por los Comités la coordinación de la actualización de las Recomendaciones de Práctica Clínica previamente elaboradas por los mismos, convocando, de acuerdo con sus autoridades, a otros miembros de su Comité y de la SAD. Con la excepción que plantea la situación actual de creación de esta función, cada Comité de Trabajo designaría en el futuro su representante ante el Comité Editorial en la instancia de renovación de sus autoridades.

Dentro de nuestros próximos pasos, les informamos sobre la creación de una asesoría en metodología de la investigación, financiada con recursos propios, que brindará servicios en forma gratuita bajo la condición de publicar con obligatoriedad los resultados de la investigación en la Revista de la
SAD. Asimismo se está trabajando en la elaboración de un listado de referentes voluntarios para tareas de asesoría académica quienes, en principio, se sumarán a las tareas del futuro Comité Editorial. Como fuente obligada, y muy importante de trabajos originales, recordamos a los receptores de subsidios de la SAD que deben enviar sus trabajos con obligatoriedad a la Revista lo más pronto posible dentro del año de finalización del trabajo a fin de cumplir cabalmente con las condiciones iniciales de otorgamiento.

Hemos descrito la situación actual de la publicación y nuestros próximos pasos. Necesitamos hacerlo juntos, y por tal motivo convocamos a todos los miembros de la SAD para que consideren el envío de trabajos originales de investigación en diabetes tanto básicos, como clínicos y epidemiológicos. De este modo, con la colaboración de todos, lograremos que continúe el progreso en la calificación internacional de la Revista para alcanzar y superar las metas propuestas.

\section{Directores del Comité Editorial de la Revista de la Sociedad Argentina de Diabetes}

